\title{
CARCINOMA OF THE PENIS
}

\author{
By Milroy Paul \\ Professor of Surgery, University of Ceylon
}

Carcinoma is the gravest disease affecting the penis. There are, however, some redeeming features. All cases are curable if they are treated before the growth has metastasized to the inguinal glands. The disease can be entirely prevented if circumcision is practised in infancy.

The disease is not a common one. Sir James Paget and Mr. Sibley (Jacobson, I893) reckoned that I per cent. of all cancers occurred at this site and $\mathrm{McCrea}$ (I940) gives similar figures (I to 3 per cent.). An interesting feature of the disease is its varying incidence in other parts of the world. Ngai (1933) found that the incidence in China was I 8.3 per cent. and in other Far Eastern countries it was 18.9 per cent. By contrast the incidence in Continental Europe was 4.9 per cent., while it was only 2 per cent. for Great Britain and the United States of America. The incidence in India was worked out by Wolbarst (1932) from available reports in the literature to be 15.9 per cent. of all cancers in male subjects. In Ceylon the writer ( 1929 ) found that of $4 I_{5}$ cases of malignant disease coming to the General Hospital, Colombo, 55 were cases of carcinoma of the penis ( 13.2 per cent.) Cooray (1944) found that of 2,293 biopsies in Ceylon, one-third were carcinoma of the penis. These figures suggest a progressive increase in the incidence of the disease as one passes eastwards from America to the Far East.

There are other features of interest in regard to the incidence of the disease. Carcinoma of the penis in all countries affects males of the poor classes. The well to do are almost immune. Circumcision plays an important part in influencing the incidence of the disease. Wolbarst (1932) found that the cancer of the penis does not occur in Jews who had been circumcised in infancy. There had been no authentic case on record. The disease had been recorded in an uncircumcised Jew, suggesting that the Jew enjoys no racial immunity to the disease. Amongst Mohammedans, who practice ritual circumcision between the fourth and the ninth years but not before, cancer of the penis does occur but it is a rare occurrence. An interesting conclusion has been drawn from this. Circumcision in infancy prevents the disease from occurring at all, but circumcision in later childhood while also protective, cannot altogether prevent carcinoma occurring in middle life. In these cases there would be precancerous changes which would be latent for many years. Dean (1949) had treated over 30 cases with a penile cancer who had been circumcised 40 or more years before the onset of the disease. Penile cancer is, however, exceptional in the circumcised. It is a disease occurring almost exclusively in the uncircumcised, particularly in those with phimosis.

In Great Britain and the United States of America, cancer of the penis is a disease affecting males between 50 and 70 years, while in countries in which the incidence of this growth is higher, the age of onset is lower, viz. 35 to 50 years. Venereal diseases play no part in influencing the incidence of cancer of the penis.

Carcinoma of the penis should more properly be designated carcinoma of the preputial sac, for the growth always arises within this sac either on the inner surface of the prepuce or on the glans penis. Are there any special features making the preputial sac a favourable site for the development of cancer? The prepuce has a secretion peculiar to itself, the smegma preputii, arising from the glands of Tyson on the corona and neck of the penis. The smegma is a secretion of fatty material (Lehmann, 1925) and its chemical composition suggests that like certain hydrocarbons it has carcinogenic properties. Smegma imprisoned behind a non-retractile prepuce moistened with stagnant urine could cause chronic irritation and this might, in time, lead to the development of a cancer. In most cases of carcinoma of the penis there are, however, no detectable precancerous changes in the preputial sac. There is one precancerous state occurring in the preputial sac which is readily recognizable. This is the condition of leucoplakia which presents precisely the same appearances as those of leucoplakia of the tongue or of the buccal mucous membranes. When the change affects the mucosa of the prepuce in a case of phimosis, the orifice of the prepuce becomes further constricted and there is recognizable scarring at the orifice. There may even be obstruction to micturition. Should a cancer develop within such a preputial sac it would be latent for a considerable time. Attention might be drawn to the possibility of a cancer having developed from the presence of a foul discharge from the preputial orifice, and there might even be an indurated area 
palpable within the preputial sac. In the later stage the growth might fungate through the preputial skin or through the orifice of the prepuce. If the leucoplakic change were limited to the mucosa of the prepuce, a circumcision would rid the patient of the diseased focus, and if a cancer had just commenced at this site such a circumcision would suffice to cure the patient of his cancer. The ordinary method of circumcision carries the risk of cutting through cancer tissue with the possibility of implantation of cancer cells in the circumcision wound. The writer uses a method for circumcising these cases which avoids this risk. A circular incision is made in the coronal plane through the preputial skin $I$ in. in front of the corona. Retraction of the penile skin proximal to this incision exposes the outer surface of the mucous membrane of the prepuce. A small snick into the preputial sac is made through the mucosa of the prepuce near the corona and the opening so made is enlarged by continuing to cut through the mucosa of the prepuce at its junction with the glans penis. The whole of the mucosa of the prepuce is thus excised and even if there were a cancer on the mucous membrane it would have been removed entire. Leucoplakia also affects the epithelium covering the glans penis. If the glans were covered by a non-retractile foreskin, the leucoplakia would not be visible and a cancer developing on it might be latent. An offensive discharge from the preputial orifice or an induration within the preputial sac should necessitate wide opening of the preputial sac for visualization of the glans penis. When leucoplakia affects the glans penis only, a bold decision has to be made. The patient is well advised to submit to a partial amputation of the penis, even when there is as yet no evidence of cancer. A biopsy could not serve to reassure such a patient that a cancer had not already commenced, as the biopsy might not section the area of the cancer. Retention of a glans penis afflicted with leucoplakia carries the high probability that cancer would ultimately occur at this site. Partial amputation of the penis is the only measure combating this risk with certainty of success.

Cancers of the penis arising from a leucoplakia accounts however for only i I per cent. of the cases. The common papillary carcinoma accounts for 50 per cent. of cases, the nodular for 29 per cent. and the infiltrating carcinoma for ro per cent. These types of carcinoma are readily recognizable provided that the affected area is not concealed by a phimosis. The papillary carcinoma is an exuberant cauliflower-like mass. It remains a surface growth for a long time and will eventually metastasize to the lymphatic glands before it invades local tissues. The nodular type and the in- filtrating type invade the sheaths of the corpora cavernosa and this could lead at a later stage of the disease to the dissemination of metastatic nodules within the copora cavernosa. A remarkable feature of cases of carcinoma of the penis is the rarity of involvement of the urethral orifice. A patient with a massive fungating growth of the penis will be able to micturate freely through the growth owing to non-involvement of the urethral orifice.

The treatment of carcinoma of the penis is much concerned with the question whether the growth would be better treated by radium therapy or by amputation of the penis. Radium therapy conserves the penis and if it were an effective method of treatment it should replace treatment by amputation of the penis. Carcinoma of the penis is a squamous cell carcinoma. On 'a priori' grounds it should be very suitable for treatment by radium. Unfortunately carcinomatous growths in the penis are often radio resistant. Windeyer (1939) found that only the hypertrophic and the ulcertative types were radio sensitive. Ngai (1939) found that the proliferative type belonged to Broder's grade I and II, the nodular type to Broder's grade II and III and the infiltrative type to Broder's grade III and IVQ Stanford Cade (1939, 1948) views the problem differently. He advocates radium therapy for the early case and amputation of the penis for the late case. The question is, however, coloured by the responsibility of the medical attendant to his patient. Amputation of the penis will cure all the early cases and many of the late ones. Radium treatment might fail to cure the early case, and as long as the position is as uncertain as this it is more reasonable to operate on all cases except those who refuse an amputation of the penis. Amputation of the penis will rid the patient of his primary growth and local recurrence is almost unknown after this operation. On the other hand, even if the growth has already metastasized to the inguinal glands, operation will save the patient from the miseries of fungation of the primary growth. Moreover, radium treatment is not without complication. Persistent painful ulceration from radio necrosis may necessitate amputation of the penis. Atrophy of the testis has occurred from the effects of radium applied to the primary growth.

Amputation of the penis is attended with one chief difficulty-the successful management of the urethra (Pearce Gould, 1882). The unsuccessful case develops an intractable stricture at the urethral orifice which needs a re-amputation of the urethra at a higher level for its relief. Galbraith (1949) wrote, 'stenosis of the urethral meatus is a common complication which may be avoided by careful 
and neat suturing of the urethra. The writer has found, however, that all methods in which the skin is sutured to the urethral orifice are liable to be complicated by a stricture, even those in which the urethral orifice had been enlarged by mucosal flaps. The urethra shrinks, drawing in a cone of skin, and a stricture often develops at the apex of this cone of skin. Mr. Hilton separated the corpus spongiosum from the corpora cavernosa and left it projecting beyond the skin. The writer has found that this is the only way of ensuring a permanently patent urethral orifice. The corpus spongiosum is dissected away from the corpora cavernosa and it is divided well forwards so as to permit of a stump of urethra $\frac{3}{4}$ in. long projecting beyond the level of the skin. With this method the patient micturates without difficulty from the time of the operation, and although the actual urethral orifice bears no sutures no stricture develops at this site.

The choice between a partial and a total amputation of the penis is determined by the possibility with the former operation of conserving a penile stump which would project $\frac{1}{2}$ in. beyond the peno-scrotal junction. If this cannot be done a shorter stump would lead to dribbling of urine over the scrotum, which would be more troublesome and inconvenient than micturating from the perineal urethra provided by the total amputation. A partial amputation of the penis would give the most satisfying result if it conserved the maximum length of penis consistent with the necessity for making a radical excision of the growth. The circular amputation of the penis most nearly complies with these requirements, for it can be performed almost up to the level of the margin of the growth, as the growth does not involve the skin much beyond its visible margins and because it also does not extend within the corpora cavernosa much beyond the external margins of the growth. Amputations of the penis utilizing skin flaps to cover the distal end of the penis do not conserve its maximum length, and the 'raison d'etre' for making a skin flap, the placement of the urethral orifice away from the line of the skin suture, does not possess any advantage if the urethral stump is projected $\frac{3}{4}$ in. beyond the surface of the skin.

The circular amputation incision is conveniently performed in the following manner. A circular incision is made through the skin in the vertical plane immediately proximal to the growth. The penile skin proximal to the skin cut is retracted towards the root of the penis exposing the corpora cavernosa and the corpus spongiosum. The corpus spongiosum is divided as far forwards as the growth would permit and it is dissected off the corpora cavernosa proximally for $I$ in. The corpora cavernosa are cut across at this point, bleeding being controlled by digital compression of the base of the penis. Suture of the sheaths of the corpora cavernosa in a vertical line will arrest haemorrhage from its cut surfaces. A rubber tourniquet is not needed and is best avoided as it is clumsy and interferes with the manipulation necessary to obtain the maximum length of penis. The skin is sutured vertically with the urethra projecting beyond the skin incision for $\frac{3}{4}$ in.

The operation of total amputation of the penis is rightly ascribed to Pearce Gould (1882), for although total amputation had previously been practised by Thiersoh (1877) and Davies Colley, Pearce Gould was the first to introduce the method of detaching the roots of the corpora cavernosa off the bone. It is unnecessary to split the scrotum to gain access to the attachments of the corpora cavernosa as advocated by Pearce Gould. An incision is made in the perineum, over the urethra, and the corpus spongiosum is isolated and divided across $I$ in. beyond its junction with the membranous urethra. The corpora cavernosa are detached from the ischiopubic rami by sharp dissection, bleeding vessels being tied. The proximal stump of the corpus spongiosum is now dissected and drawn out of the perineal wound and anchored by sutures with $\frac{3}{4}$ in. of stump projecting beyond the skin surface. The rest of the perineal skin wound is sutured. A circular incision of the skin of the penis at the peno-scrotal junction will expose the corpora cavernosa and the corpus spongiosum at this place. Division of the suspensory ligament of the penis and of the dorsal vein of the penis will allow of the penis with the corpora cavernosa being removed intact. The circular skin incision is sutured vertically with a rubber drain in it leading backwards to the perineum.

Involvement of the inguinal glands by cancerous deposits is a grave complication in a case of carcinoma of the penis. It marks an abrupt transition from the case which is curable to the case which is well nigh incurable. Radical excision of the lymphatic glands in both groins, including Cloquet's gland in the femoral canal, will be followed by a painful solid oedema of both lower limbs and scrotum, bringing the patient to a condition in which death would be a merciful release. Excision of only the obvious hard, painlesslyenlarged lymphatic glands would not be a rational operation, but it is probably the best compromise in the circumstances. Deep ray therapy and radium therapy have proved to be ineffective in the control of metastases in the inguinal glands. Involvement of the external iliac glands places the patient in a group in which recovery cannot be expected. In the later stages of the disease fungation of cancerous deposits in the inguinal glands adds considerably to the misery of the patient. 
Carcinoma spreads from the penis to the inguinal glands by lymphatic embolism. This is fortunate, for the operation of amputation of the penis can, in consequence, completely excise the primary growth and thus secure the patient from any risk of local recurrence. If the inguinal glands have not been involved the operation would cure the patient, but if the inguinal glands are already involved the operation would at least save the patient from the horrors of a fungating growth of the penis.

\section{BIBLIOGRAPHY}

CADE, S. (1939), Proc. R. Soc. Med., 33, 1509.

COORAY, G. H. (1944), Indian F. Med. Res., 32, 71 .

DEAN, A. L. (1950), 'A Text Book of Surgery,' edited by Christopher. W. Saunders \& Co., Philadelphia, p. 131 r.B.
DAVIES COLLEY, cited by Pearce Gould.

GALBRAITH, W. W. (1948), "Textbook of Genito-Urinary Surgery,' edited by Winsbury White. E. \& S. Livingstone, Edinburgh, p. 609 .

HILTON, cited by Pearce Gould.

JACOBSON, W. H. A. (1893), 'The Diseases of the Male Organs of Generation.' J. \& A. Churchill, London, p. 707

LEHMANN, cited by Ngai.

MCCREA (1940), 'Diseases of the Urethra and Penis.' John $\stackrel{\vec{F}}{\rightarrow}$ Wright \& Sons, Ltd., Bristol, p. 27 r.

NGAI, S. K. (1933), Amer. F. Cancer, 19, 259.

PAUL, M. (1929), Fournal of the Ceylon Branch of the Brit. Med. Assoc., 26, No. 2, p. 13.

PEARCE GOULD, A. (1882), Lancet, May 20, 821 .

THIERSOH, cited by Pearce Gould.

WOLBARST, A. L. (1932), Lancet, i, I 50.

WINDEYER, B. W. (1939), Proc. R.'Soc. Med., 32, 1504 की

WINSBURY' WHITE, H. B. (1948), "Textbook of GenitoUrinary Surgery.' E. \& S. Livingstone, Edinburgh.

THOMSON-WALKER, J. (I936), 'Genito-Urinary Surgery.' Cassel and Company, 'Ltd., London.

\title{
SIMPLE OBESITY
}

\author{
By John S. Richardson, M.V.O., M.D., F.R.C.P. \\ Physician, St. Thomas's Hospital
}

The interest that our forebears had in obesity was social rather than medical. It was displayed in its more flamboyant form by the voluptuaries of some of the ruling houses of Europe and painters of the School of Titian and Rubens, who delighted in mastering the shadows cast by massive folds of flesh. It also appealed to the sporting instincts of the inhabitants of this country who love records that can be numerically expressed.

The present desire of most people to avoid obesity reflects not only an alteration in taste and the present dictates of fashion, but also shows an awakening to some unpleasant realities. Insurance statistics, largely those of the Metropolitan Life Insurance Company of America, show that between the ages of forty-five and fifty for every Io $\mathrm{lb}$. overweight there is roughly a ro per cent. increase in the death rate over the average for that age. This is largely a result of cardiovascular and renal disease, whilst fat diabetics have a very much poorer outlook than thin. Age certainly contributes to the incidence of these disorders in the obese, but the evidence of the dangers of obesity is overwhelming. The problem is, therefore, quite literally a vital one.

\section{Physiological Considerations}

Physiologists have tackled it for many years from numerous aspects and a great deal of work has been done. Nevertheless, it is fair to say that up to now all metabolic studies have failed to shof why some get enormously obese and others remain slim all their lives. It seems clear that in the obese food is no less efficiently absorbed than in the normal. It is not converted into fat or stored more easily, neither is it mobilized less easily, nor burnt more economically. Recently Dodds (I950) reported some experiments by which he has examined this subject. He found that when some people whose weights have been constant over many years were given double or treble their normal intake, they did not put on weight. $\mathrm{He}$ showed that in them this was not due to a failure of assimilation or digestion, but suggested that some studies that were being made in his department on their respiratory metabolism might show that they responded to a high caloric intake by an increase in their metabolic rate. The control series of those who got fat with this gross overfeeding showed no such increase in metabolism. These observations call to mind the theory of 'Luxus Konsumption'.(Grafe and Graham, I9II; N Grafe, 1933) which has long been in disrepute, $N$ Wiley and Newburgh (193I) finding no evidence 은 to support it. Dodds (1950) made the novel $\omega$ suggestion that the mechanism that keeps most people's weight so remarkably constant was the $\stackrel{\varrho}{\leftarrow}$ gravitational pull of the earth, but he has so far $\mathbb{D}$ been unable to devise experiments to test his hypothesis. 